\title{
Extending Life in Duchenne Muscular Dystrophy: Implications for Appraisals of Cost-Effectiveness
}

\author{
Erik Landfeldt ${ }^{1}$ (]
}

Published online: 26 November 2018

(c) The Author(s) 2018

In recent years, several new treatments have been developed, or are currently tested in trials, for Duchenne muscular dystrophy (DMD), a rare, X-linked recessive, severely debilitating, and ultimately fatal neuromuscular disorder characterized by progressive muscle wasting, multi-system complications, and a mean life expectancy of about 25 years $[1,2]$. Although involving different therapeutic mechanisms, these interventions generally aim to reduce, halt, or reverse the rate of muscle degeneration, thereby delaying time to key disease milestones, including loss of independent ambulation and the need of ventilation support for survival [2]. As a consequence, there is reason to believe that these drugs for DMD may also have a non-trivial impact on life expectancy through, for example, further stabilized cardiac and respiratory functioning.

However, despite being of obvious benefit and value to affected patients, including efficacy data on mortality in economic evaluations of drugs for DMD may prove challenging. The reason is two-fold. First, DMD is associated with chronic care needs and is thus a very costly disease to society. This is particularly true for advanced stages of DMD, where patients start to experience more pronounced impairment of their upper extremities and eventually need assistance with all basic and instrumental activities of daily living [3]. Second, due to the multi-dimensional impact of the disease on patients' health, quality of life in DMD is typically found to be relatively poor as quantified in terms of utilities representing general population preferences for health states. For example, the mean annual direct cost of illness and patient utility in non-ambulatory adults with DMD in the US have been estimated at about \$54,000 and 0.18, respectively [3].

Erik Landfeldt

erik.landfeldt@ki.se

1 Institute of Environmental Medicine, Karolinska Institutet, Nobels väg 13, 17177 Stockholm, Sweden
In cost-utility analysis, where the incremental cost of an intervention is related to the incremental gains in terms of quality-adjusted life-years (QALYs), these two aspects mean that treatments that prolong survival in DMD will be associated with only a modest benefit (i.e. a low QALY gain), but at the same time a substantial cost. Indeed, continuing the example above for the US, extending life for a patient with DMD by 1 year would be associated with a QALY gain of 0.18 at a cost of $\$ 54,000$ (not accounting for the cost of the evaluated intervention), resulting in an incremental costeffectiveness ratio of $\$ 300,000$, well above both conventional and heavily extended cost per QALY willingness-topay thresholds in most jurisdictions. Put differently, although of obvious humanistic and clinical value, improving prognosis for survival in DMD is not expected to be cost-effective.

While ethical and health economic aspects of this phenomenon have been discussed in the literature, the focus has been on end-of-life treatments indicated for patients with a short life expectancy, for example end-stage cancer drugs [4]. However, as illustrated above, challenges of including efficacy on mortality in cost-utility analyses are certainly also relevant to treatments targeting chronic diseases associated with extensive care needs and poor quality of life, such as DMD. This information should be helpful to inform the interpretation and contextualization of outcomes from economic evaluations in this indication. Furthermore, the examples provided imply that adjusted criteria, comparable to those implemented by the National Institute for Health and Care Excellence (NICE) in the UK for evaluations of end-of-life treatments indicated for patients with a short life expectancy [5], may be appropriate also when testing the cost-effectiveness of drugs extending life in DMD. Yet, additional research is also needed to further examine the public's views on the relative value of life-extending treatments, as well as therapies targeting rare as opposed to common illnesses, to help align health policy with the preferences of the general population. 


\section{Compliance with ethical standards}

Funding None.

Conflict of Interest Dr. Erik Landfeldt conducts research for pharmaceutical companies marketing treatments for DMD.

Open Access This article is distributed under the terms of the Creative Commons Attribution-NonCommercial 4.0 International License (http://creativecommons.org/licenses/by-nc/4.0/), which permits any noncommercial use, distribution, and reproduction in any medium, provided you give appropriate credit to the original author(s) and the source, provide a link to the Creative Commons license, and indicate if changes were made.

\section{References}

1. Birnkrant DJ, Bushby K, Bann CM, Apkon SD, Blackwell A, Brumbaugh D, et al. Diagnosis and management of Duchenne muscular dystrophy, part 1: diagnosis, and neuromuscular, rehabilitation, endocrine, and gastrointestinal and nutritional management. Lancet Neurol. 2018;17(3):251-67.

2. Crone M, Mah JK. Current and emerging therapies for duchenne muscular dystrophy. Curr Treat Options Neurol. 2018;20(8):31.

3. Landfeldt E, Lindgren P, Bell C, et al. The burden of Duchenne muscular dystrophy: an international cross-sectional study. Neurology. 2014;83:529-36.

4. McHugh N, Baker RM, Mason H. Extending life for people with a terminal illness: a moral right and an expensive death? Exploring societal perspectives. BMC Med Ethics. 2015;16:14.

5. The National Institute for Health and Care Excellence (NICE). Appraising life-extending, end of life treatments. https://www. nice.org.uk. Accessed Aug 2018. 